\title{
HYPERCOLLAPSED NUCLEAR MATTER
}

\author{
Y. NE'EMAN \\ University of Tel-Aviv, Tel-Aviv, Israel, and University of Texas, Austin, Tex., U.S.A.
}

\begin{abstract}
We evoke the possible existence of hypercollapsed tightly bound nuclear isomers, due to the existence of an attractive 'heart' deep inside the repulsive core.
\end{abstract}

\section{Introduction}

Physical theory has yielded in the past a series of predictions relating to new states of matter, or to novel types of material constituents. Successful predictions have included new chemical elements, previously unknown elementary particles, the world of antiparticles, etc. and recently macroscopic nuclear matter in the form of neutron stars. As yet unfound are magnetic monopoles, quarks, $W$ mesons; superheavy elements and collapsed material in 'black holes' (or coming out of 'white holes'). To this list we are now adding the hypothesis of the existence of hypercollapsed nuclear matter.

\section{Repulsive Core and Attractive Heart}

We were led to the first suggestions of the existence of such tightly bound nuclear isomers by considering the new evidence about a strongly attractive internucleon force at very short ranges, beyond the barrier of the repulsive core (Ne'eman, 1968a, b). It is this region that we shall name the 'attractive heart' inside the repulsive core.

The evidence for the attractive heart comes from high energy physics. Internucleon forces - and not just in two-particle (four-prong) processes - can be represented by the exchange of Regge trajectories (Collins, 1971). For forward and near-forward scattering these are meson trajectories; backward scattering is produced by the exchange of two nucleons. The meson trajectories appear in a Chew-Frautschi plot as straight lines: the spin is linear in $M^{2}$ for the various meson states lying at positive momentum-transfer squared $t^{\prime}$ at integer $J$, either even or odd.

The quark model suggests that there should be trajectories passing through states with $(J=$ the spin; $P=$ the space parity; $C=$ the charge parity of the neutron component)

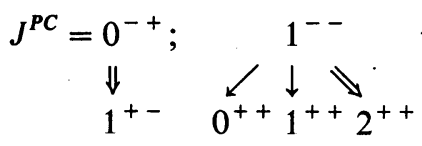

where the upper row corresponds to the two $s$-wave quark-antiquark combinations. The recent results of duality theory confirm the observation of exchange-degeneracy, which connects $0^{-+}$to $1^{+-}$and $1^{--}$to $2^{++}$(our double arrows).

Experimentally, all of these mesons are now known (Söding et al., 1972) except for 
the $0^{++}$set which is as yet uncertain. For reasons of covariance, the $1^{++}$set does not play an important role in the near-forward region. For zero-strangeness cases, we have two isoscalar and one isovector state for each $J^{P C}$; this would imply dealing with five states per $J^{P C}$, i.e., 30 states altogether (or 18 isospin $\times J^{P C}$ multiplets). Actually, the isoscalar $\mathrm{O}^{-+}$is relatively weakly coupled to the nucleon, because of the peculiar $F / D$ ratio in the SU(3) coupling. In the $1^{--}, 0^{++}, 1^{++}$and $2^{++}$the particular form of SU(3) breaking mixes isoscalars so that only one is coupled to the nucleon. This leaves us with the following (Collins, 1971, with masses in parentheses),

$\begin{array}{lcccrr} & 0^{-+} & 1^{+-} & 1^{--} & 0^{++} & 2^{++} \\ I=1 & \pi(140) & B(1235) & \rho(765) & & A_{2}(1310) \\ I=0 & \eta^{\prime}(960) & & \omega(785) & & f(1260)\end{array}$

Phenomenologically, the couplings of the $2^{++}$are about five times stronger (in $g^{2} / 4 \pi$ ) than the $1^{--}$couplings (Michael, 1970). This implies an attractive heart which should have some chance of producing a second potential well, closer to the nucleon's center.

The existence of a tensor force in nuclei has been known for a long time. The new high-energy picture points to the possibility that aside from quadrupole moments, the longitudinal component of a $2^{++}$exchange might create an attractive heart - depending of course upon the particular many-body situations arising for various baryon numbers. This is plausible, since the elementary interactions, repulsive and attractive, are of the same order of magnitude, and it is only the variation in $A$ and in configurations which can establish clear cut regions of preponderance.

From the experimental evidence it now looks as if Regge trajectories keep rising indefinitely in the Chew-Frautschi plot. This has been idealized in duality as a system of straight lines (e.g., the Veneziano model), perhaps an overstatement. However, it should at least provide for a further hard core reappearing at even shorter range. This would help in creating conditions in which the hypercollapsed state would be stable, rather than tend to collapse further if external pressure is applied.

\section{Nuclear Isomers}

Bodmer (1971) has studied the possible existence of hypercollapsed nuclei in terms of the nuclear Hamiltonian. He assumes this Hamiltonian to contain in addition to the 'normal' $H_{A}(N)$, a part $H_{A}(C)$ for the collapsed version corresponding to the same baryon number $A$.

Before going into any details, it is instructive to consider the analogy with molecular (nuclear) fusion and fission. Taking a deuterium molecule (the $N$ state), we know that it is fusion-favored, i.e., that given the right conditions it can 'collapse' into a He atom (the $C$ state); the $D-D$ system is originally bound by $B_{2,2}(N)<B_{2,2}(C)$ the binding energy in He. On the other hand, at the other end of the periodic table we have fissioning nuclei (or 'molecules'), i.e., with the fission products originally bound by $B_{A}(C)<B_{A}(N)$, the binding in a pseudo molecule made of the fission-product atoms. 
The radius of the hypercollapsed state $R_{\mathrm{c}}<R_{\mathrm{N}}=r_{0} A^{1 / 3}$ and is either a constant ( $\lesssim 0.5 \mathrm{fm}$ ) or a saturating radius $R_{\mathrm{c}} \sim r_{\mathrm{c}} A^{1 / 3}$ with $r_{\mathrm{c}}<0.4 \mathrm{fm}$. As in the above molecular examples, the most general possibility would correspond to at least one cross-over region $A_{\text {crit }}$, but the requirement of spherical symmetry at the microscopic level appears to invert the sequence:

$$
\begin{array}{lll}
B_{A}(C)<B_{A}(N) & \text { for } A<A_{\text {crit }} \\
B_{A}(C)>B_{A}(N) & \text { for } A>A_{\text {crit }} .
\end{array}
$$

Bodmer estimates $A_{\text {crit }}$ to lie somewhere between $A=16-40$. To reach this value, he treats the second case; assuming the lifetime of a 'normal' stable nucleus to be $\tau_{A}(N)>10^{31} \mathrm{~s}$ (one collapse per mole per year), he estimates the penetrability of the saturation barrier (the hard core) $W(r)$ between normal and collapsed states. Comparing $\tau_{A}(N) \geqslant 10^{31} \mathrm{~s}$ with the 'period' $\tau$ corresponding to $R_{A}(N)$, i.e., about $10^{-22} \mathrm{~s}$, he gets $\left(P\right.$ is the penetration probability, $\tau^{-1}$ the number of penetration 'attempts' per second)

$$
P=10^{p} \leqslant 10^{-22} \div 10^{31}=10^{-53} \text {. }
$$

Using various plausible values for the nuclear parameters does not modify $p$ by more than $20 \%$. $P$ can now be related to the equation of state of nuclear matter - which is of course part of our guess. Thus

$$
p=\exp \left\{2\left(2 M / \hbar^{2}\right)^{1 / 2} \int_{R_{\mathrm{c}}}^{R_{\mathrm{N}}}[W(r)]^{1 / 2} \mathrm{~d} r\right\},
$$

where $M \approx A m_{\mathrm{N}}$ is the mass of $N_{A}$, the normal nucleus. It is by extrapolating smoothly from the region around $R_{\mathrm{N}}$ where we know the equation, to the region of a hypothetical $R_{\mathrm{c}}$, that Bodmer gets his estimate of $A_{\text {crit }}$.

For the binding energy per nucleon in the hypercollapsed state, we have to assume that it will stay in the hundreds of $\mathrm{MeV}$, so as to avoid zero or negative energy nuclei.

\section{Unitary Symmetry}

The Fermi energy may rise to $0.5 m_{N}$ or above. This will then lead to the creation of hyperons and mesons. In the extreme case, we might have the unitary spin equivalent of an $\alpha$ particle: $A=16$, with all eight octet baryons appearing with spins up and down. The next 'complete shell' would be at $A=56$, with all components of 8 and 10 etc. The total strangeness (and electric charge) tends to be very low in general (and 0 in the 'complete' cases). Bodmer gets similar results from a rough quark model calculation.

\section{5. 'Superbaryons' and Cosmology}

In recent years, a thermodynamical approach to strong interactions (Hagedorn, 1970) has been used to predict results of high energy multiparticle collisions in accelerators 
and in cosmic rays, and to study the early stages of the expanding universe. This approach provides, it seems, an efficient way of accounting for the strong interactions through the insertion of an infinite spectrum of metastable ('elementary') states, which implies there is a maximal temperature to hadrons. In this approach, the development of concentrations (leading to the ultimate formation of galaxies) appears to be inescapably tied up with the existence of 'superbaryons.' A superbaryon is a quasi-elementary particle, with

$$
10^{67}>A>1 \text {. }
$$

It is required (Hagedorn, 1972) that the decay of a galactic-mass state go through cascade emission of nucleons with the large-A state, appearing as a hadron. Ordinary nuclei do not fit the above description; hypercollapsed nuclear matter might be just what is required.

Note that as an energy source, if $B_{A}(C) \gg B_{A}(N)$, we might have a new mechanism in the gradual collapse of normal to hypercollapsed matter.

\section{Observation}

The search for hypercollapsed matter resembles the search for quarks, in that we are after unusual $e / M$ ratios (here because of new $M$ values instead of $e$ values). Cosmic rays appear to be one possible place to search; present limits on nuclear isomers (as pointed out by W. Webber in a private communication to $\mathrm{H}$. Kasha) do not get under $5 \%$. Neutral collapsed matter would be more difficult to find. One could also search material on the Earth's crust (though hypercollapsed matter might sink gravitationally).

\section{Astrophysics}

In the main, the existence of hypercollapsed nuclei would modify present estimates of the size of neutron stars, etc. We would have a new region of stability, following white dwarfs and 'normal' neutron stars. Neutron star material would also include dense 'raisins' of hypercollapsed matter. Further catastrophic collapse into a black hole - a possibility raised by the effects of $2^{++}$attraction in the center of the neutron star, i.e. superstrong short-range gravitation - would be inhibited by the existence of a new repulsive barrier as predicted by the Chew-Frautschi plot (the $\rho$ or $\omega$ Regge recurrences).

\section{Acknowledgments}

The author is indebted to Dr R. Hagedorn for an illuminating discussion, and to Dr H. Kasha for an enlightening correspondence.

\section{References}

Bodmer, A. R.: 1971, Phys. Rev. D4, 1601.

Collins, P. D. B.: 1971, Phys. Reports 1C, 103.

Hagedorn, R.: 1970, Astron. Astrophys. 5, 184. 
Hagedorn, R.: 1972, lecture given at the Ettore Majorana School of Astrophysics and Cosmology. Michael, C.: 1970, Springer Tracts Mod. Phys. 55, 174.

Ne'eman, Y.: 1968a, in Science Year, World Book Science Annual, Field Enterprises Educationa. Co., Chicago, p. 157.

Ne'eman, Y.: 1968b, in A. Perlmutter et al. (eds.), Symmetry Principles at High Energy, W. A Benjamin, New York, p. 149.

Söding, P., Desy, J. B., Barbara-Galtieri, A., Enstrom, J. E., Lasinski, T. A., Rittenberg, A., Rosenfeld, A. H., Trippe, T. G., Barash-Schmidt, N., Bricman, C., Chaloupka, V., and Roos, M.: 1972, Phys. Letters 39B, 1.'

\section{DISCUSSION}

Bethe: I do not understand why it is not possible for a few nucleons to go into the condensed state, if that state exists, rather than the entire nucleus. I can well understand that $t w o$ nucleons would not have a condensed state, for the same reason that a deuteron has such a small binding. But four nucleons should be very favorable, just like the alpha-particle, and in this case the penetrability should be very high - perhaps $P=10^{-10}$. Then with the characteristic nuclear time of $10^{-22} \mathrm{~s}$, the lifetime of an ordinary nucleus against collapse would be $10^{-12} \mathrm{~s}$ - clearly unacceptable. If nuclear forces again saturate at these smaller distances, i.e. if $R_{\mathrm{c}}=r_{\mathrm{c}} A^{1 / 3}$, then one should except the quasialpha-particle to have essentially the full binding energy per particle for a collapsed nucleus, because for $A=5$ the p-shell would have to be started, with much diminished binding. Therefore I believe the estimate of $10^{-12} \mathrm{~s}$ for a collapse time is reasonable.

If, on the other hand, there is no saturation, i.e. if the radius of collapsed nuclei is $R_{\mathrm{c}}=r_{\mathrm{c}}$ independent of $A$, then it might be necessary to assemble a large number of nucleons before the collapsed state becomes energetically favorable, and then the long collapse times might be realized.

$N e$ 'eman: I agree with the general spirit of your comment which is one of the reasons I mentioned a lower bound, i.e. a minimal baryon number for the collapsed state to appear. This is indicated by its non-appearance in abundance in the form of partly-collapsed heavier nuclei, in which an internal alpha-particle would have collapsed. I think however that your figure of $10^{-10}$ for the penetrability of the particle is somewhat too large, being based upon the Coulomb barrier rather than upon the forces we have in this case.

The no-saturation picture for the collapsed state might indeed be more plausible because of the non-appearance of collapse for a small number of baryons.

Bethe: If a collapsed state for nuclei exists, this would have a catastrophic effect on neutron stars. Namely, if neutrons are brought together within a distance of order $r_{c}=0.4 \mathrm{fm}$ by external forces, i.e. by gravitation, then the collapsed state would automatically be formed, without the need of going through a potential barrier. In other words, the equation of state would be very soft. It would not be possible to form neutron matter of a density corresponding to $r_{0}=r_{\mathrm{c}}$ which is four nucleons $\mathrm{fm}^{-3}$. In fact, the pressure would have a maximum at a much lower density than this, because at $r_{\mathrm{c}}$ the energy is supposed to be negative. This would keep the maximum density of a neutron star way below $6 \times 10^{15} \mathrm{~g} \mathrm{~cm}^{-3}$, and the maximum pressure correspondingly low. Accordingly, the maximum mass of a neutron star would be very low, and the maximum moment of inertia much below $2 \times 10^{44} \mathrm{~g} \mathrm{~cm}^{2}$ which is the most probable (minimum) value for the Crab pulsar.

$N e$ 'eman: If indeed we should take the value of $2 \times 10^{44} \mathrm{~g} \mathrm{~cm}^{2}$ for the moment of inertia it seems to put a lower bound on the mass of a neutron star. Counting a binding energy of $50 \mathrm{MeV}$ per nucleon in the collapsed state should not make a large difference in our estimate of the star's mass, but its size will of course be 2-3 times smaller. Considering that we do not have independent knowledge of the star's baryon number, we do not know if a size of $5 \mathrm{~km}$, for instance, corresponds to the number of baryons in one solar mass at normal nuclear density, or to perhaps 10 times as many baryons at collapsed distances and a density which is larger than normal even though it is smaller than at intermediate distances.

Bethe: Concerning observations, I understand that cosmic ray physicists, e.g. Dr Peters at Copenhagen, can observe both charge and mass. If only nuclei like ${ }^{16} \mathrm{H}_{\mathrm{e}}$ (which should exist if some of the nucleons are converted into hyperons) were to exist, it would stand out very clearly and should be discoverable in much smaller quantities than $5 \%$.

Ne'eman: I agree that the 'hyperonized' types of collapsed nuclei would be easier to discover. However, the entire question of the abundances of such nuclei makes it difficult to get good upper bounds for cosmic rays. 